Théologiques

Théologiques

\title{
Dieu et la scène liturgique
}

\section{Guy Lapointe}

Volume 6, numéro 2, octobre 1998

Dieu interdit

URI : https://id.erudit.org/iderudit/024963ar

DOI : https://doi.org/10.7202/024963ar

Aller au sommaire du numéro

Éditeur(s)

Faculté de théologie de l'Université de Montréal

ISSN

1188-7109 (imprimé)

1492-1413 (numérique)

Découvrir la revue

\section{Citer cet article}

Lapointe, G. (1998). Dieu et la scène liturgique. Théologiques, 6(2), 61-72.

https://doi.org/10.7202/024963ar

\section{Résumé de l'article}

Dans la liturgie, la réalité de Dieu existe; elle est presque de l'ordre d'une évidence. Cette réalité de Dieu est mise en scène. Pourtant, dans cette expérience, le réfèrent Dieu reste toujours la réalité non manifeste, abstraite, qu'on appelle à « venir en présence » et que le rituel propose de manifester concrètement. Que signifient les diverses mises en scène liturgiques qui ouvrent l'espace d'une relation à Dieu et à l'autre? Jusqu'à quel point la liturgie est-elle vraiment un lieu de révélation et de mémoire? Le temps dans la célébraiton liturgique n'indique-t-il pas, par la mise en scène, le passage de Dieu dans la vie? A cet égard, l'eucharistie reste ce lieu paradigmatique d'une parole et d'un geste que libère le renoncement à toute possession de Dieu et de l'autre. d'utilisation que vous pouvez consulter en ligne.

https://apropos.erudit.org/fr/usagers/politique-dutilisation/ 
Théologiques 6/2 (1998) 61-72.

\title{
Dieu et la scène liturgique
}

\author{
GuY LAPOINTE \\ Faculté de théologie \\ Université de Montréal
}

La liturgie doit garder la beauté du geste inachevé.

Bernard Feillet

Un poète doit laisser des traces de son passage, non des preuves.

Seules les traces font rêver.

René Char

Poser la question de Dieu, surtout en regard de la scène litur gique, n'est-ce pas forcer en quelque sorte une évidence? Comment peut-on simplement imaginer que Dieu pourrait ne pas être l'acteur incontournable de toutes nos liturgies? Pourtant, si dans beaucoup de nos célébrations, l'encombrement des rites, même simplifiés au courage de Vatican II, ne fait qu'ajouter à l'encombrement de nos relations aux autres, Dieu est toujours aussi fortement affirmé et confessé. La scène liturgique apparaît à plusieurs, comme une vieille médaille, au profil presque tout effacé, mais dont on sait que, une fois au moins, il a été célébré et prononcé comme un mot plein de sens. Le mot «Dieu » existe; il est mis en scène.

Qu'on soit croyant ou pas, ce mot incite à un travail d'intelligence, jusqu'à devenir une question qui touche aux enjeux les plus profonds des êtres humains et même les plus décisifs pour leur destin. Une vigilance s'impose cependant, qui appelle à ne pas se tromper de dieu et sur Dieu. D'autant que, dans la scène liturgique, Dieu est perçu comme le référent dont on ne peut parler qu'avec beaucoup de difficultés. En cette expérience, le référent est «la réalité non manifeste, abstraite, fuyante, dont la présence est à jamais 
incertaine, que le rite propose de manifester concrètement... ${ }^{1}$ ». D'où le questionnement que je fais mien : porter la question de Dieu en théologie et en liturgie, qu'est-ce à dire? Tel sera mon premier temps de réflexion. J'aborderai ensuite l'expérience liturgique à partir de trois axes: la révélation, la mémoire et le temps. Je terminerai par quelques notes sur la référence à Dieu dans l'eucharistie.

\section{Dieu dans la théologie et dans la liturgie}

Depuis saint Anselme, et bien avant lui, la théologie, dans les meilleurs moments de sa tradition intellectuelle, s'est surtout définie comme une "fides quaerens intellectum". C'est précisément dans cette appréhension d'une foi en recherche d'intelligence que la perception d'un Dieu dont le nom seul est "sauf ${ }^{2}$ ", a pu faire droit à la naissance du discours théologique. On a cherché alors la question de Dieu là où elle se posait : dans les interrogations des humains, leurs angoisses, leurs révoltes, leurs constructions symboliques et rituelles, tous lieux d'appel à une transcendance et à passer, en quelque sorte, de l'autre côté.

Au contraire, lorsqu'on a conçu la théologie comme "la science de Dieu ", le risque était grand que Dieu devienne l'objet possédé, livré à la joute des dogmes, et que, dans la polyphonie des discours constitués, ce discours en vienne à n'apparaître, à plus d'un, difficilement pensable dans la modernité, pas plus qu'il n'est habitable. Le peu de crédibilité que semble retenir le discours théologique dans le monde universitaire vient, en bonne partie, d'une élaboration épistémologique trop souvent confondue avec une apologétique de la Vérité confessée, avec une telle arrogance parfois, qu'elle n'arrivait plus à solliciter le respect des autres approches scientifiques si ce n'est, le plus souvent, qu'en leur résistant. Comme tout travail intellectuel le réclame, les théologiens ont appris, à leurs dépens, que leur discipline se singularise davantage en ce qu'elle aborde la question de Dieu non comme une affirmation de Dieu, mais comme un dis-

1 Ph. OLIVIERO et T. OREL, «L'expérience rituelle », RechScRel 78/3 (1990) 337.

2 Je fais ici allusion au texte de Jacques DERRIDA, Sauf le nom. Paris, Galilée, 1993. 
cours sur le problème de Dieu, même si ce discours se situe dans une tradition croyante.

S'il apparaît un luxe que de se poser la question de Dieu jusqu'à prétendre la porter au statut de discours universitaire parmi les autres disciplines, comment est-ce possible, dans le cadre de ce débat interdisciplinaire autour de l'inter-dit Dieu, de rendre compte des diverses mises en scène de Dieu dans les liturgies? À cet égard, on est certainement redevable aux recherches d'Emile Durkheim qui ont réussi, en un véritable renversement copernicien, à montrer que les conduites rituelles et leurs mises en scène n'étaient plus reléguées à l'enfer des aberrations humaines, mais qu'elles prenaient place désormais parmi les objets "sérieux " de connaissance et d'attention $^{3}$. Louis-Marie Chauvet, s'appuyant sur les recherches ethnologiques, de même que sur les autres sciences humaines et principalement les sciences du langage, a initié une recherche et un développement théologiques, pour donner d'autres appuis à une conception d'ensemble de la symbolique chrétienne ${ }^{4}$. Le travail sur les rites chrétiens qu'Augustin avait commencé et qui fut poursuivi par $S$. Thomas fut, avec Chauvet, repris en modernité.

Mais au-delà de cette conceptualisation liée à l'anthropologie durkheimienne et à sa prise au sérieux du phénomène rituel, la question de Dieu reste intacte pour le théologien liturgiste. Où est Dieu dans les liturgies qui s'adressent à lui dans une kyrielle de nominations? À quoi ressemble Dieu dans les liturgies chrétiennes, pour nous en tenir principalement à cette tradition? C'était déjà la sorte d'interrogation que portait Isaïe : " $\grave{A}$ qui donc avez-vous laissé ressembler Dieu? Quelle image en avez-vous instituée » $(40,18)$ ? N'est-ce pas pure illusion que de continuer à célébrer Dieu dans toutes les liturgies "inventées" par les grandes traditions religieuses, depuis des temps immémoriaux? Il importe de ne pas oublier - ce que déjà saint Augustin évoquait- que la forme extérieure du culte et l'inté-

3 Émile DURKHEIM, Les formes élémentaires de la vie religieuse: le système totémique en Australie. Paris, P.U.F. 1985 (première édition, Alcan, 1912).

4 Louis-Marie CHAUVET, Symbole et Sacrement. Une relecture de l'existence chrétienne. Paris, Cerf (Cogitatio Fidei 144), 1987. Les sacrements. Parole de Dieu au risque du corps. Paris, Les Editions Ouvrières, 1993. 
gration théologique ou même éthique de l'expérience de Dieu, n'a jamais été de possession tranquille en christianisme. Ce fut l'objet d'un débat permanent.

En fait, pour beaucoup, Dieu est là dans les liturgies, présent, possédé, mais souvent sans l'interrogation que porte Augustin :

Voulez-vous savoir si Dieu est là? Quand vous vous toumez vers lui, avez-vous la sollicitude du genre humain? ${ }^{5}$

Pourtant, on se retrouve à participer à des mises en scène dans lesquelles la place de Dieu soulève d'immenses interrogations à la fois sur les figurations de sa présense-absence, sur sa nomination, en même temps que sur une certaine appropriation de Dieu dans ce lieu symbolique. $\grave{A}$ cet égard, la liturgie est-elle un espace symbolique pour « laisser passer " Dieu, dont, pour reprendre l'expression de René Char, "seules les traces font rêver», ou n'est-elle pas surtout un lieu d'auto-manifestation d'un groupe religieux qui finit par « se fabriquer » son dieu? Le cas de la célébration de l'eucharistie est exemplaire d'un jeu liturgique qui a fini par façonner un imaginaire croyant qui a gommé l'absence de Dieu pour en faire une présence pleine à travers le Christ, éliminant même ou presque le geste de fraction du pain qui exprimait, à même la mémoire du geste de Jésus à la Cène, le jeu d'une absence-présence de Dieu. Geste d'un Dieu imprenable, rencontré dans le regard et le geste de la main tendue de l'autre.

Un des apports récents dont ont profité la théologie et la pratique chrétienne, c'est l'émergence de l'élément symbolique de la foi, relié à la dimension symbolique de l'être humain. Cette dimension pourrait être caractérisée comme l'espace non conceptuel à la source du connaître, comme le champ de non-maîtrise qui est au fondement de toute appropriation. Le symbole tente de suggérer, par quelques mots - souvent des récits - par des gestes, des personnes et des objets, ce qui est au-delà du dit, du faire, voire du réel, du moins au niveau limité où nous pouvons saisir celui-ci. Le symbole met en valeur la dimension invocatrice du langage, qui s'adresse avant de dire; son dire est plus de l'ordre de l'évocation que de la définition; les gestes faits sont plus de l'ordre de l'incantation que de la production; les 
choses indiquent la Chose que personne ne peut produire, mais qui peut-être se donne.

La liturgie est le champ et le cadre d'une relation à Dieu, avonsnous l'habitude de dire. $\grave{A}$ même cette relation, la liturgie devrait provoquer un élan symbolique entre les membres qui célèbrent. C'est une assemblée en état d'invocation. À l'intérieur de cette communauté symbolique, paroles et gestes ont d'abord valeur invocatrice, appel adressé à Dieu et aux humains. Ils ont aussi valeur évocatrice, c'est-à-dire qu'ils suggèrent, reproduisent la grande symbolique de Dieu à même l'humanité, objet non d'abord de connaissance, mais de récits, de témoignages et de foi; ils ont enfin valeur d'incitation éthique dans la mesure où ils ouvrent un nouvel horizon du monde, ils reposent la question du comment apprendre à vivre dans la recherche de l'autre et de Dieu.

On est alors surpris de ce paradoxe qui se dessine dans la pratique liturgique : alors que la mise en scène est adresse à Dieu, faite de conduites cérémonielles, cet espace devrait être vécu comme une dépossession, une dé-prise, alors qu'il est souvent, à l'insu des célébrants eux-mêmes, confiscation, possession de Dieu. On revient toujours à cette expérience des disciples d'Emmaüs racontée en Lc 24 : le Dieu de Jésus ne se donne plus à voir et à croire que dans les symboles de son absence et ici, en l'occurrence, dans le partage du pain et de la coupe en mémoire de Lui. De même que le discours théologique aborde la question de Dieu, non comme une affirmation de Dieu, mais comme un problème de Dieu, l'instance liturgique, elle, se caractérise par le jeu de l'adresse, de l'appel, de la confession et de l'affirmation de foi, à travers un parcours symbolique.

\section{La liturgie, révélation, mémoire et temps}

En prenant pour acquis que la liturgie se joue à même un ensemble de configurations, un espace symbolique ouvert, un jeu de circonscriptions et de franchissements, j'aimerais suggérer en quoi elle est un lieu-transit. La liturgie est une mise en scène qui tente de dessiner un mouvement d'adresse à Dieu, à même notre propre dramatique humaine. Elle est à la fois le paradigme du passage de Dieu dans la vie et de sa non-appropriation par un groupe, en même temps qu'elle est espace du passage de Dieu. Dans l'Évangile, on entend Jésus dire à ses disciples : "il vaut mieux pour vous que je m'en aille » 
(In 16,17), comme pour laisser une empreinte plus marquée des traces de Dieu. Mais c'est aussi une invitation à se rendre compte qu'il n'existe pas de « rencontre » avec Dieu pour qui s'établit hors de luimême. N'est-ce pas «l'autre » qui se tient à l'intérieur, si l'on peut dire, et l'intérieur est une place où se tenir, ou mieux un carrefour, comme on l'entend chanter aux hymnes pascales de Patrice de la Tour du Pin ${ }^{6}$. Cette mise en scène touche aux sources les plus élémentaires de toute foi en Dieu et de toute théologie : la révélation, la mémoire et le temps. Ce sont ces trois éléments, qui façonnent la prise ou la dé-prise de Dieu, et que j'aimerais reprendre par quelques remarques.

\subsection{La symbolique de la révélation}

L'espace liturgique est, avons-nous dit, ouverture, adresse à Dieu. Elle est vécue comme un jeu symbolique, comme le lieu d'une révélation. Révélation de Dieu aux croyants et révélation des croyants entre eux. Révélation de quoi? de qui? On sait que dans la tradition judéo-chrétienne, la compréhension de la révélation de Dieu dans la vie du peuple, et dont les écrits bibliques en sont les empreintes, reste un lieu de grands débats. Toute l'histoire d'Israël, à même ses migrations, se recueille dans des moments de célébration. Ses liturgies célèbrent à la fois une manifestation de Dieu et s'ouvrent en bénédictions et en prières. Ces liturgies, dans un jeu de figurations, ne font que raconter les passages de Dieu, depuis la création jusqu'à l'apocalypse. Tous ces dispositifs symboliques, mis en scène, ne résistent qu'à «faire croire » à l'autre et à maintenir, persistante, l'interrogation: mais qui es-tu? Comme autant d'inscriptions dans des monuments et dans des temps sacrés, comme autant de gestes inachevés, ces liturgies en s'adressant à l'autre, référent invisible, sont toujours des espaces d'attente d'une révélation et peut-être d'une réponse à la question, qui mettrait fin à l'interrogation et à toute liturgie.

Cette attente d'une révélation de Dieu est aussi et surtout révélation sur nous. Comme en un jeu de places, la liturgie nous renvoie à notre propre questionnement en regard de nous-mêmes et des autres et de l'Autre. La liturgie ne parle-t-elle pas plus facilement de nous 
que de Dieu. Elle nous met dans les dispositions d'attente, d'accueil. Qu'est-ce qui est révélé? De quelle nouveauté s'agit-il? Est-ce nouveauté ou ce qui, de toujours, était déjà là - est déjà là - et qu'on ne voit pas, qu'on cache ou dissimule? Dieu ne révèle-t-il que notre nouveauté toujours à venir, toujours en retard d'être. La liturgie comme mise en scène d'une révélation permet une lecture par après, une post-compréhension de ce qui a été interprété et de ce qu'on pourrait interpréter comme des passages ou des traces de Dieu laissées au cœur des événements. Pour reprendre une expression de Derrida, n'est-elle pas un «post-scriptum ". La liturgie révèle le chemin de révélation de Dieu dans ce monde; elle ouvre l'horizon de l'être humain dans sa recherche de lui-même et de Dieu. Confesser Dieu dans la liturgie, n'est-ce pas du même coup se tourner vers l'autre, là où il est question de Dieu, là où Dieu fait signe, se signale, se signe. Jeu de l'offrande, dans un présent où se " fait la vérité ".

"Dieu, toi que nous n'avons jamais vu, et que nous trouvons cer tains jours plus absent que présent, nous savons que tu es là plus proche que lointain.... " Autant de paroles, à la fois prière et bénédiction, adressées au Dieu "plus absent que présent ". La révélation est toujours, d'une certaine façon, d'" ailleurs", comme Dieu, comme nous. C'est au jeu de l'altérité que nous sommes appelés dans la liturgie et nous prions, en quelque sorte, pour nous ouvrir les uns aux autres, pour que l'autre ouvre notre monde. Pour y arriver, la liturgie, dans ses jeux de configurations symboliques, doit accomplir son travail de nouveauté, de révélation. Elle nous incite à ne pas se tromper de Dieu, à ne pas se tromper sur Dieu. Pas plus que sur nousmêmes.

\subsection{La mémoire dans l'espace liturgique}

Si la scène liturgique permet un espace de révélation sur ce qui est toujours là, de quoi alors permet-elle de faire mémoire? Dans la liturgie chrétienne, cette mémoire est marquée par les événements qui vont de la création jusqu'à l'eschatologie, tous ces événements étant colorés par celui du passage de Jésus. Ce «faire mémoire " devient alors ouverture sur une histoire, un fait de salut non à la manière de quelque chose simplement oublié, qui pourrait être à nouveau présent, rendu présent, mais de la mémoire d'un passage. La liturgie célèbre le passage de Jésus, en fait mémoire, mais ne le 
retient pas. Cette mémoire célébrante renvoie à un immémorial dont le souvenir ne restera toujours qu'une trace.

À cet égard, la liturgie célèbre un présent qui est tout à la fois passage, qui ne vit pas de présence donnée, ni antérieure et à retrou ver, ni future ou à anticiper, mais qui vit des traces qu'ont laissées en nous les passages liés à l'infini de nos présents, passés et dépassés. Or trop souvent, l'instance rituelle favorise non le passage de Dieu en révélation et en mémoire, mais devient, dans le jeu symbolique, un lieu pour transformer Dieu en une présence et une mémoire contrôlables.

La liturgie est un jeu symbolique de faire mémoire et de faire retour, de mémoire étrange de l'essentiel : laisser passer Dieu à travers les événements célébrés. C'est à ce point du "laisser aller ", que la liturgie pourrait retrouver sa capacité de susciter le désir. Francis Guibal écrit qu'il "faut perdre le discours assuré de la parole vive, de la présence pleine, du réel sans faille ${ }^{7} »$. Il est difficile de consentir à laisser s'échapper absolument les signes que nous traçons, seule façon d'apprendre à les envoyer, d'un envoi risqué, jamais sûr d'arriver, d'un envoi d'amour: "Pas de don sans oubli absolu, oubli de ce que tu donnes, à qui, pourquoi, et comment, de ce que tu t'en rappelles et espères. Un don, s'il y en a, ne se destine plus ${ }^{8}$ ».

Parce qu'elle ne risque plus le passage et l'envoi, parce qu'elle retient les signes tracés à même sa tradition, la liturgie est peu créa trice de mémoire. Par manque de mémoire, la communauté devient abstraite; elle s'évanouit. Une pratique de la mémoire qui réduit l'écart à une présence pleine, masque l'altérité, puisque l'autre est sommé de revenir au même. Dieu revenu au même, otage de nos espaces symboliques. Alors, la mémoire ne peut plus fonctionner, parce qu'elle se transforme en souvenir de choses passées ou d'êtres passés. Dieu au passé et au futur et non Dieu de passage.

7 Francis GUIBAL, Autonomie et altérité. Paris, Cerf, 1993, p. 134.

8 Jacques DERRIDA, La carte postale de Socrate à Freud et au-delà. Paris, Flammarion, 1991, p. 85. 


\subsection{Le temps dans la liturgie}

Les liturgies épousent toujours et très fortement la couleur du temps. On n'a qu'à penser à l'expression " les temps liturgiques ", utilisée dans la tradition chrétienne, et qui permet des périodisations qui nous situent et situent nos tâches. En un certain sens, les liturgies sont cycliques en ce qu'elles reviennent à des moments très précis, comme pour nous laisser en présence d'une conscience vive du temps. Qu'est-ce que le temps, sinon la conscience que les êtres comme les choses passent? et que non seulement nous passons, mais que nous sommes toujours, en quelque sorte en retard d'être, par rapport aux choses qui passent. Et Dieu? Dieu a-t-il créé, crée-t-il et recrée-t-il dans le temps? Dieu crée en ce qu'il y a passage, naissance et accès à soi. Le temps dans la célébration liturgique participe de la mise en scène et de la prise de conscience, à la fois individuelle et commune, du passage de Dieu dans la vie et dans notre aménagement du temps et des " effets" de ce passage sur la recherche de sens de nos vies.

C'est en cela que la liturgie joue, rassemble, re-cueille les croyants, à certains moments de la vie, comme en un mouvement de communauté, pour ouvrir ces moments à l'interrogation sur le sens de la vie et sur l'aventure de Dieu. Naissance, union, mort, mémoire de la résurrection du Christ, sont autant de temps liturgiques qui façonnent la mémoire de foi et qui peuvent devenir des moments de révé lation. Mais, la célébration de l'eucharistie joue le rôle de paradigme dans ce jeu liturgique ou mémoire, révélation et temps en constituent les dimensions essentielles.

\section{L'eucharistie : un geste poétique}

Le partage d'un repas touche au plus intime de la condition socio-culturelle. La gamme de ses aspects symboliques et matériels est quasi-complète. Le rituel religieux, les complicités ou les confrontations, les discours graves ou ludiques, la mémoire des origines et de la fin, les révélations des autres et des dieux, et les temps de la vie, sont les grands axes de cette expérience fondamentale de l'existence humaine. C'est la convivialité, le "convivere ", le vivre avec et parmi d'autres dans la forme la plus articulée et chargée, qui est celle du repas partagé. 
N'est-ce pas à travers et à même ces gestes humains de partage et de mémoire, de révélation aussi, que se découvre quelque chose du visage de Dieu. N'est-ce pas ce que Jésus a voulu vivre juste avant de mourir : repas d'adieu, lié à l'amitié et à la trahison, microcosme de sa vie et de la vie de tout être humain. C'est en se référant à ce repas d'adieu, au seuil de la fête du "Grand Passage », geste poétique s'il en est un, que les croyants tentent d'ouvrir leur propre itinéraire de vie à la révélation de Dieu, à même ce geste de mémoire, de partage et d'action de grâce. Dans cet acte liturgique, la mise en scène est révélatrice de ce que, dans les gestes accomplis par les croyants et les autres, Dieu apparaît dans les traces que laisse la mémoire partagée en pain et en vin. Telle une métaphore pour dire la trace de Dieu qui a marqué le monde d'une façon décisive, dans la personne de Jésus. N'est-ce pas cela qui est à lire et que toutes les générations interprètent depuis ce soir-là et continueront d'interpréter, souhaitant garder vivante la mémoire en même temps que la question sur Dieu?

Ce geste, repris en eucharistie, à la lumière de la Pâque, laisse ouvert les voies de l'imaginaire qui permet à ce qui est vrai d'échapper à l'enfermement de toutes les compromissions de la vie. Il s'inscrit dans le champ de la conscience et y rejoint l'espace sans limite de l'espérance. Cet instant n'appartient ni au passé, ni au présent, ni à l'avenir. Le temps ne peut le revendiquer pour l'inscrire dans son irréfragable déroulement. Nous échappons alors à la mesure du temps pour communier, en dépassant les âges de la vie et la définition des jours, à l'affirmation de l'être et pourquoi pas de Dieu.

Et si ce geste de partage donne à quelqu'un d'être présent à lui même, attentif à la présence de l'infini en toute existence, il ouvre la présence du Christ à la présence de Dieu. La présence qui se révèle ici est celle de l'être humain au milieu de son être en com. munion au mystère de Dieu qui l'habite. C'est ainsi que, étonnés du dépouillement de cet acte, nous prenons qu'un peu de pain pour en laisser à l'autre. Depuis longtemps, nous sommes « avertis "par une sorte d'intuition de la foi, que nous ne pouvons pas saisir Dieu dans cet espace que libère le renoncement à toute possession. Alors seulement, grandit la mémoire de ce que fut Jésus, lui dont l'évocation réveille en nous le désir d'accomplir notre vie avec les autres. Une formule de Bernard Feillet me paraît bien exprimer la force et la fragilité de ce geste : "...Célébrer l'eucharistie c'est choisir de se tenir nu devant Dieu et, dans une attitude de confiance sous le regard de 
l'autre, accepter d'être nu à ses yeux ${ }^{9}$ \%. En ce geste, il nous est demandé d'être là tout simplement - pourquoi passer tant de temps, dans nos liturgies, à s'avouer pécheur?- pour renoncer à l'impossible désir de la pureté de Dieu, seule façon de nous disposer au possible de notre vie. Cela suffit à célébrer la présence de Dieu. Je retrouve ici une autre formule de Feillet: « La présence eucharistique, c'est la présence de l'homme à lui-même et au mystère de Dieu dans son être ${ }^{10}$ \%.

En fait, la communion et le partage requis de l'assemblée qui célèbre l'eucharistie manifestent que la présence de Dieu est entre les participants et participantes. Elle ne peut se saisir indépendamment des êtres en présence. Les symboles du pain et du vin, lorsqu'ils retrouvent un geste significatif de partage, expriment cet insaisissable présence. Cette présence ne peut qu'être ressentie comme immense. À cet égard, c'est le consentement à cette immensité de la présence de Dieu comme de celle des autres, qui donne à la vie de s'ouvrir à la révélation de l'Autre, au temps qui passe et qui nous façonne, mais aussi à la mémoire ou au souvenir d'un avenir. Laisser passer Dieu à même la présence à l'autre dans le souci de l'autre à même la communion au pain et au vin. Le geste de tendre la main dessine la trace de la présence de l'Autre et à l'autre.

\section{Conclusion}

Le risque de la liturgie, c'est que le rituel donne l'illusion d'un espace rempli où il est possible d'avoir la mainmise sur Dieu, sur les autres et sur soi. S'il en est ainsi, ce rituel-là serait destiné à la consommation, don sans réciprocité, sans appel au désir de l'autre, sans mouvement vers l'autre. La liturgie, au contraire, est lieu et espace symbolique de l'autre, regard vers l'autre, attention à l'autre, adresse à l'Autre; elle est le lieu de l'appel, de la venue de la pré sence à l'absent. Elle me renvoie à ma responsabilité de faire mémoire dans le temps qui passe. Lieu d'altérité, la liturgie - et, de façon paradigmatique, l'eucharistie - demeure, en un certain sens, un lieu intenable qui nous force à rester sur la corde raide de la vie et du

9 Bernard FEILleT, L'Errance. Paris, Desclée de Brouwer, 1997, p. 62.

10 Ibid., p. 65. 
rapport à autrui. La liturgie, loin d'apparaître comme le lieu de nos sécurités, interroge la vie et Dieu et pourquoi pas! peut aussi intégrer le temps du doute qui nous permet de porter la question. À cet égard, on peut reprendre une expression de Eric-Emmanuel Schmitt, l'auteur de la pièce Le Visiteur, et l'appliquer à toute personne et même à toute assemblée qui entre dans l'espace liturgique : "Je doute en Dieu, non en dehors 11 ». La liturgie n'est-elle pas, à ses heures, un espace capable de permettre tout autant la confession de foi que le " doute en Dieu "?

11 Éric-Emmanuel SCHMITT a fait la chronique "L'invité " dans L'Actualité religieuse, 14 février, 1997, p. 41 